\title{
Liver Pathology
}

Editor

JOHN HART

\section{SURGICAL PATHOLOGY CLINICS}

www.surgpath.theclinics.com

Consulting Editor

JASON L. HORNICK

June 2018 • Volume 11 - Number 2 